\title{
About the influence of friction and polydispersity on the jamming behavior of bead assemblies
}

\author{
L. Pournin ${ }^{1, a}$, M. Ramaioli ${ }^{1}$, P. Folly ${ }^{2}$, and Th.M. Liebling ${ }^{1}$ \\ 1 Mathematics Institute, École Polytechnique Fédérale de Lausanne - EPFL, CH-1015 Lausanne, Switzerland \\ 2 armasuisse, Feuerwerkerstrasse 39, CH-3602 Thun, Switzerland
}

Received 16 March 2007

Published online: 14 June 2007 - C EDP Sciences / Società Italiana di Fisica / Springer-Verlag 2007

\begin{abstract}
We study the jamming of bead assemblies placed in a cylindrical container whose bottom is pierced with a circular hole. Their jamming behavior is quantified here by the median jamming diameter, that is the diameter of the hole for which the jamming probability is 0.5. Median jamming diameters of monodisperse assemblies are obtained numerically using the Distinct Element Method and experimentally with steel beads. We obtain good agreement between numerical and experimental results. The influence of friction is then investigated. In particular, the formation of concentric bead rings is observed for low frictions. We identify this phenomenon as a boundary effect and study its influence on jamming. Relying on measures obtained from simulation runs, the median jamming diameter of bidisperse bead assemblies is finally found to depend only on the volume-average diameter of their constituting beads. We formulate this as a tentative law and validate it using bidisperse assemblies of steel beads.
\end{abstract}

PACS. 45.70.-n Granular systems - 83.10.Rs Computer simulation of molecular and particle dynamics 45.70.Cc Static sandpiles; granular compaction

\section{Introduction}

Confined granular flows are known to arch suddenly about their confining boundaries under given conditions. While this may be due to microscopic interactions in fine powders, large grains will undergo jamming as well due to mechanical equilibria induced by purely macroscopic forces. This phenomenon, referred to as "arching effect", limits the ability of grain assemblies to flow. The flow regimes of powders have been extensively studied $[1,2]$. However, the case where no flow takes place has not been treated directly in those references, but merely as a limiting case in empirical flow equations. The arching effect itself has been studied in terms of the link between the jamming probability and the amount of matter flowing between the formation of two consecutive arches [3,4]. The mechanical and geometrical structures of an arch have also been studied $[5,6]$ and in particular their dependence on the packing fraction has been investigated [7]. No quantitative results exist to our knowledge about the influence of polydispersity on jamming.

With the arching effect, granular media reveal their true, unique nature by manifesting intrinsic differences with the usual states of matter. Moreover, its discontinuous, erratic nature makes the process particularly hard

\footnotetext{
${ }^{a}$ e-mail: lionel.pournin@epfl.ch
}
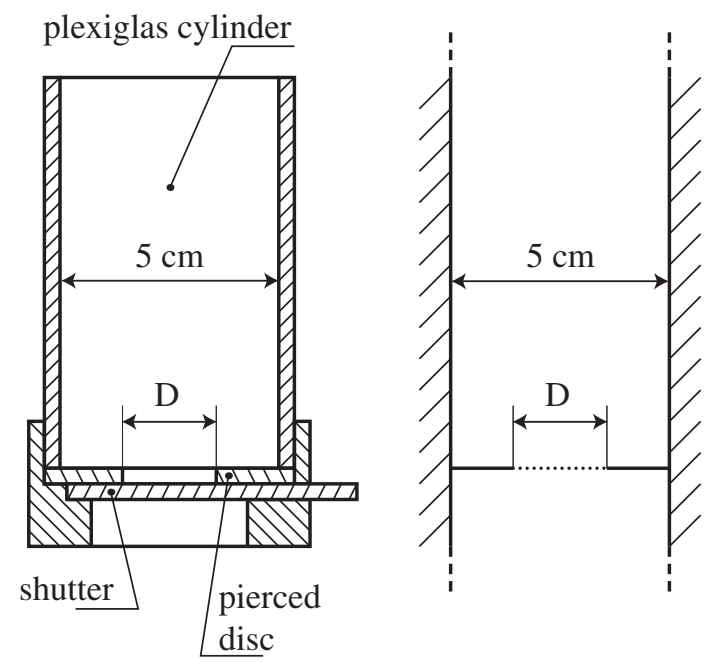

Fig. 1. The experimental setup we use to perform jamming trials (left) and its numerical model (right). The combination of the shutter with the pierced disc is modeled by a circular hole (dotted line) that can either be opened or closed.

to capture in its globality, even with stochastic modeling which has so far not been successful. This paper reports on experimental investigations of the arching effect based on numerical simulation validated with trials using 
real steel bead assemblies. The setup we study is a cylindric receptacle (Fig. 1), initially filled up with an assembly of beads that may flow out through a circular outlet placed in its bottom. Relying on a statistical characterization of the arching effect, we study the probability that an arch will form as a function of the size distribution of the beads and their friction coefficient. The formation of concentric bead rings is observed for low frictions. We identify this phenomenon as a boundary effect and study its influence on jamming. We further formulate a tentative law for bidisperse media whereby their jamming probability only depends on the volume-average diameter of their constituting beads. In addition, experimental validation confirms the simulation results, suggesting that distinct element simulation models allow for realistic reproduction of static granular phenomena.

Using the above setup, the jamming probability of a given bead assembly will be estimated as a function of the hole's diameter $D$. In this way, we can determine the diameter $\bar{D}_{j}$ of the opening hole for which the jamming probability of this medium is 0.5 . This diameter, called median jamming diameter is the quantity we use to characterize the tendency of an assembly of beads to jam.

Surface properties of the involved beads are characterized by their static friction coefficient $\mu$. This paper first reports on numerical trials conducted using the Distinct Element Method [8-11] with monodisperse bead assemblies and several friction coefficients. Experiments performed on the same monodisperse assemblies composed of steel beads show that using those numerical models with a constant friction coefficient provides realistic values for $\bar{D}_{j}$. Finally, measures of $\bar{D}_{j}$ for bidisperse bead assemblies are obtained from simulations. Those results show that for a constant friction coefficient, $\bar{D}_{j}$ only depends on the beads' volume-average diameter, which suggests that a law can be formulated about the jamming of polydisperse bead assemblies.

\section{The experiment}

The setup we use to perform jamming trials is a plexiglas cylinder with interchangeable aluminum discs at the bottom that feature a circular hole in their centers. The inner diameter of the cylinder is $5 \mathrm{~cm}$ and the bottom discs have various diameters $D$ for their central holes. The hole of the bottom disc can be obstructed using an aluminum shutter as shown on the left side of Figure 1.

The experiment proceeds as follows. First, the beads are poured into the cylinder while the hole of the bottom disc is obstructed by the shutter. The grains then pile up under the action of gravity and reach a mechanical equilibrium. Finally the shutter is suddenly pulled out and the medium either flows through the hole of the bottom disc or jams. From now on, we call $\Phi$ the bead diameter. For our experimental trials, we used steel beads with $\Phi=$ $1.0 \mathrm{~mm}, \Phi=4.0 \mathrm{~mm}, \Phi=5.0 \mathrm{~mm}$ and $\Phi=7.0 \mathrm{~mm}$. All trials were carried out using as close as possible to $0.575 \mathrm{~kg}$ of those steel beads, resulting in a filling height of around $6.5 \mathrm{~cm}$ independently of the bead diameters.
We use the Distinct Elements Method [8] to reproduce our experiment in a numerical framework. Comprehensive descriptions of the implementation we use can be found in [9-11]. Contacts are modeled using a contact time $t_{c}=6 \cdot 10^{-4} \mathrm{~s}$ and a restitution coefficient $e_{n}=0.4$ that, respectively, quantify the duration and the energy loss of simple contacts [10]. Our numerical beads have a density of $7800 \mathrm{~kg} \mathrm{~m}^{-3}$, which is that of our steel beads. The numerical model of the experimental setup is shown on the right side of Figure 1.

Numerical and experimental trials are not carried out exactly in the same way, due to their respective specificities. Observe first that the combination of the pierced disc with the shutter acts as an uneven bottom in the experimental setup while its numerical counterpart is perfectly flat. Thus, removing the shutter in experimental trials applies a shearing at the bottom of the granular piling. This is not the case in numerical trials where contacting forces suddenly disappear when the hole is opened. The numerical bead-wall friction coefficients are identical to the beadbead friction coefficient, while friction occurs between steel, plexiglas and aluminum bodies in our experiments. Finally, the initial pourings of the beads in the numerical framework and in the experimental one are different. Several methods exist to prepare numerical pilings [12]. Those methods will produce initial situations that are often difficult to achieve experimentally like spatially homogeneous polydisperse pilings. This is why we use a simplified version of the sedimentation technique [12]: we generate the beads randomly inside the containing cylinder, and move them downwards as close as possible to the bottom disc, in order to achieve a dense preliminary state. Then, we simply let the beads fall under the action of gravity from this state which is already dense enough for only few rearrangements to be needed while the initial piling settles. In particular those rearrangements will not significantly disturb the spatial homogeneity of the preliminary state in the case of bidisperse assemblies. In the experimental procedure used for monodisperse sets of beads, the pouring is done from above the cylinder instead. This would produce segregation in the case of bidisperse media.

\section{The median jamming diameter}

A trial will either result in the observation "the medium jams" or "the medium flows". We say that the medium flows if at the end of the experiment, none of the remaining beads has its center above the hole. By repeating the experiment using several pilings composed of the same beads, one can estimate the probability for them to jam with a hole of given diameter. This allows in particular to find the statistical diameter $\bar{D}_{j}$ of the opening hole for which the jamming probability is 0.5. More precisely, $\bar{D}_{j}$ is statistically evaluated as the value below which the opening diameter of flowing trials will lie with a probability of 0.5 . In order to estimate $\bar{D}_{j}$, we process the results using a generalized linear model [13]. Confidence intervals for $\bar{D}_{j}$ are found by applying a bootstrap method [13]. Note that reaching a good accuracy when estimating $\bar{D}_{j}$ 

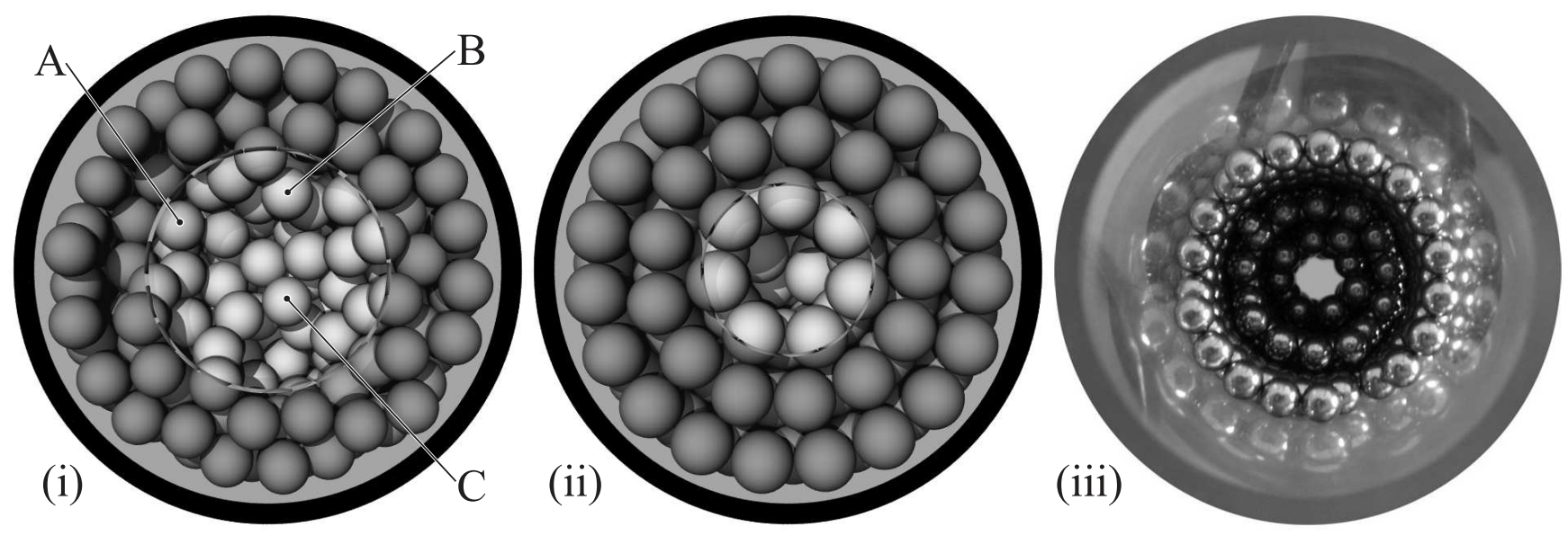

Fig. 2. Views of actual trials. Snapshots (i) and (ii) are bottom views of numerical trials where the medium jams with parameters $\Phi=6.0 \mathrm{~mm}$ and $\mu=0.8$ for (i) and $\Phi=7.0 \mathrm{~mm}$ and $\mu=0.0$ for (ii). Beads denoted A, B and C in snapshot (i) are merely held by friction. Snapshot (iii) is a top view of an experimental trial with steel beads of diameter $\Phi=7.0$ mm where the medium flows, showing the formation of bead rings. All media are monodisperse.

(i.e. confidence intervals smaller than a tenth of prediction intervals) requires between 100 and 1000 trials. The 168 numerical estimations of $\bar{D}_{j}$ reported below required some 40000 trials and around 15 years computing time on a fast single-processor machine. We carried out our actual computations on several 2.7 GHz G5 PowerPC processors and on a cluster comprising $402.8 \mathrm{GHz}$ Intel Xeon processors.

\section{Influence of friction on jamming}

The tendency of a powder to jam is known [14] to reflect the friction coefficient of its constituting particles. In our macroscopic framework, this is still the case as shown in Figure 3 for monodisperse assemblies with $\Phi=4.0 \mathrm{~mm}$, $\Phi=5.0 \mathrm{~mm}$ and $\Phi=7.0 \mathrm{~mm}$. In particular, the median jamming diameter is a globally increasing function of the friction coefficient. This dependence on friction is well marked for low values of the friction coefficient while it can practically be neglected for high ones. High frictions allow a wider range of jamming configurations than low ones because of a higher involvement of tangential forces in the stability of the system. Indeed, the possibilities for neighboring beads to carry each other are then more numerous. This results in larger arches and higher values of $\bar{D}_{j}$, explaining the trend observed in Figure 3. Inversely, low frictions yield way fewer arrangements, which are most of the time regular such as in Figure 2, (ii). Note that the values of $\bar{D}_{j}$ obtained with $\Phi=4.0 \mathrm{~mm}$ and $\Phi=5.0 \mathrm{~mm}$ are much closer for $\mu=0$ than for $\mu>0$. This will be explained below as a consequence of the formation of concentric bead rings in the container.

The median jamming diameters measured from our experiments with steel beads are shown in Table 1 and plotted in Figure 3 for $\Phi=4.0 \mathrm{~mm}, 5.0 \mathrm{~mm}$ and $7.0 \mathrm{~mm}$. These results suggest that our steel beads can be simulated with a numerical friction coefficient $\mu$ that is approximately independent of the bead size and lies between 0.2 and 0.3 .

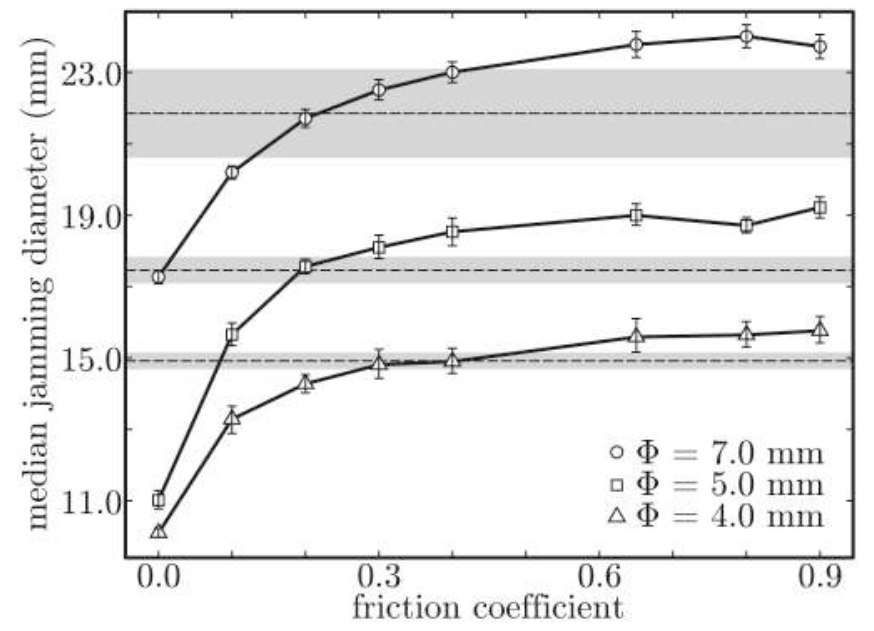

Fig. 3. The median jamming diameter obtained from numerical trials as a function of the friction coefficient for $\Phi=4.0 \mathrm{~mm}$, $\Phi=5.0 \mathrm{~mm}$ and $\Phi=7.0 \mathrm{~mm}$. Experimental values of Table 1 for $\Phi=4 \mathrm{~mm}, \Phi=5 \mathrm{~mm}$ and $\Phi=7 \mathrm{~mm}$ and their confidence intervals are reported on the graph respectively as dashed lines and greyed zones.

Table 1. Results of experimental trials on monodisperse sets of steel beads.

\begin{tabular}{cc}
\hline$\Phi(\mathrm{mm})$ & $\bar{D}_{j}(\mathrm{~mm})$ \\
\hline 1.0 & $3.88 \pm 0.15$ \\
4.0 & $14.92 \pm 0.23$ \\
5.0 & $17.46 \pm 0.37$ \\
7.0 & $21.85 \pm 1.24$ \\
\hline
\end{tabular}

This range will be discussed and refined below using experiments on bidisperse assemblies. Mind that these values certainly also reflect the differences between experimental and numerical setups and further investigations are 


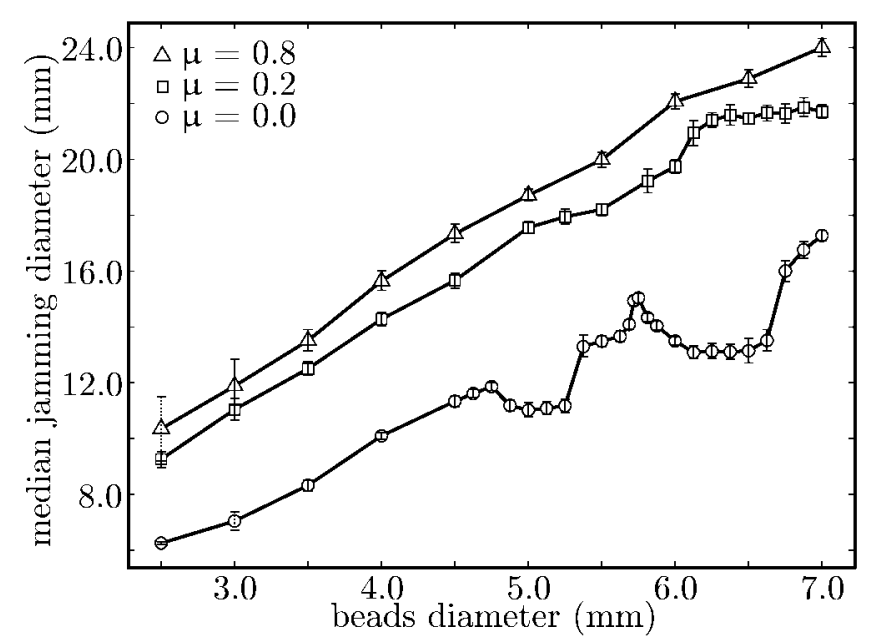

Fig. 4. The median jamming diameter plotted for simulated monodisperse media with several values of $\Phi$ and $\mu$. Due to small numbers of data for $\Phi=2.5 \mathrm{~mm}$ and $3.0 \mathrm{~mm}$ with $\mu=$ 0.0 and for $\Phi=2.5 \mathrm{~mm}$ with $\mu=0.8$, the intervals shown are prediction intervals instead of confidence intervals and are represented as dotted lines.

needed to check their actual relevance regarding the real bead-bead friction coefficient.

Friction also highly influences the shape of arches. With cohesive particles, some authors suggest that arches in two dimensions have a globally circular shape $[15,16]$. With two-dimensional dry discs, arches are always convex in the frictionless case and may be non-convex otherwise [17]. In three dimensions, the notion of convexity still has to be formally defined for granular arches. Yet, nonconvex arches are easily recognizable. In particular, most of the arches we obtain with high frictions are non-convex: they feature beads that are held by tangential forces alone, as beads A, B and C in Figure 2. With frictionless beads, almost all the arches we observed seemed to be convex.

Measuring the Coulomb friction coefficient between pairs of steel beads is very complicated. In fact the friction coefficient usually measured is that between a sphere and a plane, using a tribometer or a tilted plane. In [18], the friction coefficient was measured with both methods for steel beads yielding values between 0.1 and 0.7 depending on surface conditions. Note that this range contains the interval $0.2 \leq \mu \leq 0.3$ obtained above with our beads. As suggested at the beginning of the section, our experiment could lead to an alternate way of determining the beadbead friction coefficient. Carrying this to its maturity is a challenging subject for future work.

\section{Jamming in monodisperse bead assemblies}

Figure 4 shows $\bar{D}_{j}$ plotted against $\Phi$ for numerical monodisperse assemblies. One can see that $\bar{D}_{j}(\Phi)$ is nearly linear for $\mu=0.8$. However, for frictionless beads, $\bar{D}_{j}$ features strong local non-linear behavior given by a sequence of significant drops for $\Phi>4.75 \mathrm{~mm}$. Observe now that for $\mu=0.2, \bar{D}_{j}$ has a slight drop between $\Phi=5.25 \mathrm{~mm}$

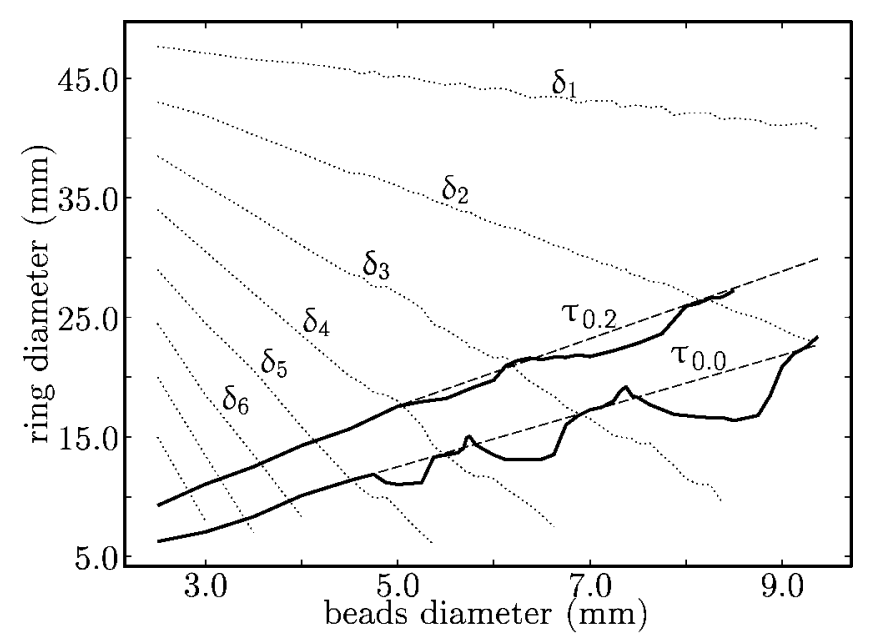

Fig. 5. Bead rings diameters $\delta_{i}$ (dotted lines) and median jamming diameters $\bar{D}_{j}$ (solid lines) plotted for monodisperse assemblies of beads. The median jamming diameters are shown for $\mu=0.0$ (lower curve) and $\mu=0.2$ (upper curve). The dashed lines $\tau_{\mu}$ show the trend $\bar{D}_{j}$ would follow for a friction coefficient $\mu$ without the effect of the bead rings.

and $\Phi=6.25 \mathrm{~mm}$ and then reaches a plateau. Irregularities of $\bar{D}_{j}$ actually also occur for non-zero friction, but for larger bead diameters than in the frictionless case, and with lower amplitudes. We identify this non-linear behavior as a boundary effect. Indeed, the cylindrical shape of the container promotes the creation of concentric bead rings as shown in Figure 2, (ii) and (iii). This effect is especially strong for large beads and low friction coefficients. Those rings disturb the linearity of $\bar{D}_{j}(\Phi)$ because their ability to flow through circular openings is not the same as that of random arrangements. Indeed, if the ring has a diameter enabling it to flow freely through the hole, it does not represent an obstacle for other beads. On the other hand, if the hole diameter is slightly below the outer ring diameter, the beads of the ring in contact with the hole will collectively reduce its diameter by approximately two bead diameters. The above-mentioned non-linearities in the plot of $\bar{D}_{j}$ against $\Phi$ nicely reflect this.

Call $\delta_{i}, i \geq 1$ the diameter of the $i$-th bead ring measured at the center of the beads and indexed from the boundary to the interior of the cylinder. At the end of each trial the bead rings appear clearly on the radial distribution of the bead centers, and the values of $\delta_{i}, i \geq 1$ can be measured precisely. We plot them in Figure 5 for $\Phi$ ranging between $2.5 \mathrm{~mm}$ and $9.5 \mathrm{~mm}$, together with the values of $\bar{D}_{j}$ obtained for friction coefficients $\mu=0.0$ and $\mu=0.2$. We observe that the ring diameters do not depend on the friction coefficient $\mu$ and that the distance between two consecutive bead rings is most of the time $5 \%$ to $10 \%$ smaller than the diameter of the beads. Some space is left empty within the rings due to the spherical shapes of their constituting beads. This can be noticed in Figure 2, (ii). Beads in adjacent rings will insert in this space in order to take advantage of the available volume in the best possible way, which reduces the distance between consecutive rings. 


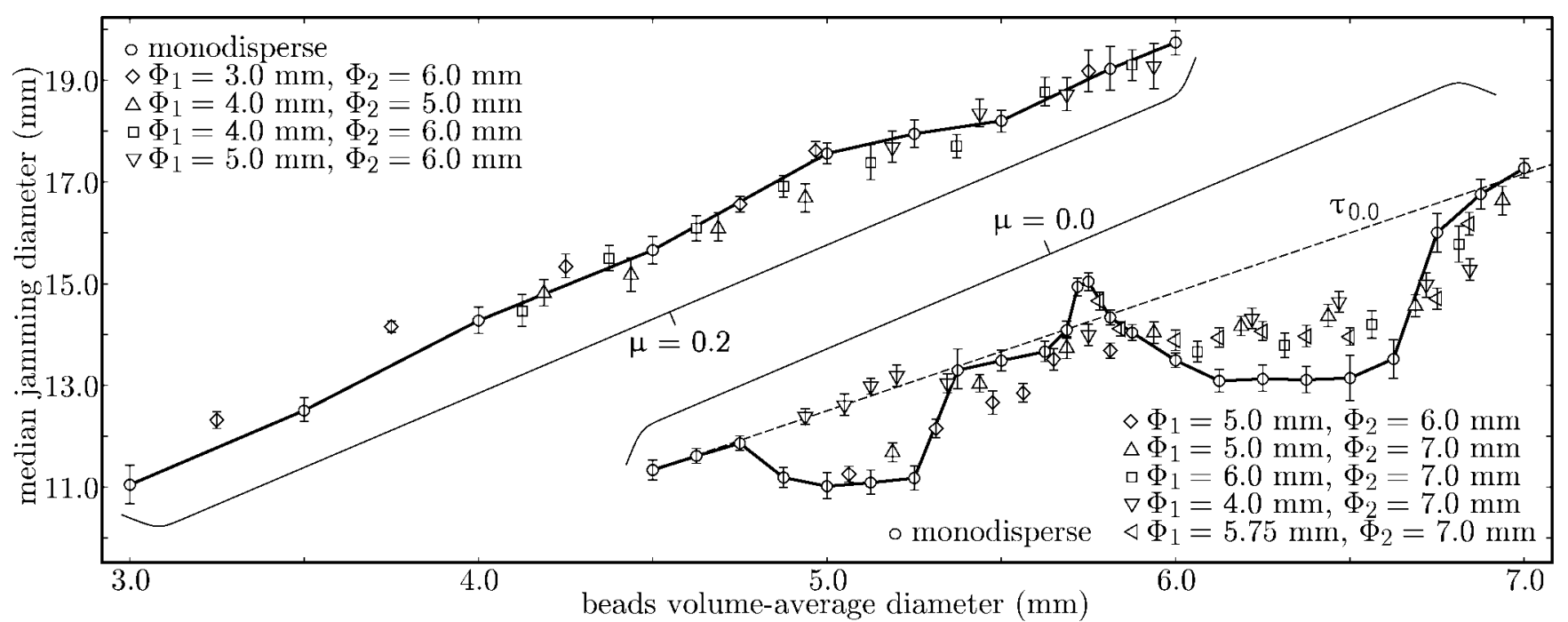

Fig. 6. Median jamming diameters obtained numerically for a selection of monodisperse and bidisperse bead assemblies. Top curve: $\mu=0.2$ and $\bar{\Phi}$ ranges from $3.0 \mathrm{~mm}$ to $6.0 \mathrm{~mm}$. Bottom curve: $\mu=0.0$ and $\bar{\Phi}$ ranges from $4.5 \mathrm{~mm}$ to $7.0 \mathrm{~mm}$.

For a given value of $\mu$, call $\Phi_{i}$ the beads' diameter so that $\bar{D}_{j}=\delta_{i}$. The points with coordinates $\left(\Phi_{i}, \bar{D}_{j}\left(\Phi_{i}\right)\right)$ lie on a same straight line $\tau_{\mu}$ (see Fig. 5). Intuition suggests that $\bar{D}_{j}$ should increase as a function of $\Phi$. This is not the case except when $\Phi$ is close to $\Phi_{i}$. Far away from $\Phi_{i}$, $\bar{D}_{j}(\Phi)$ decreases. There, the median jamming diameter is constrained to remain between two consecutive bead rings and therefore follows the decreasing trend of their diameters. It seems likely that $\tau_{\mu}$ would be the law $\bar{D}_{j}$ would follow without the effect of those periodic rings. Indeed, $\tau_{\mu}$ extrapolates to large $\Phi$ the linear behavior $\bar{D}_{j}$ has for small $\Phi$. In this light, the fact that $\bar{D}_{j}(\Phi)$ lies on $\tau_{\mu}$ in the neighborhood of $\Phi_{i}$ can be seen as a local disappearance of the ring effect.

One can see in Figure 2, (iii), that rings form in our experiments with steel beads, corresponding to a simulated friction coefficient between $\mu=0.2$ and $\mu=0.3$. This shows that bead rings also occur in reality and not only in our numerical framework and further confirms that they indeed form with non-zero-friction coefficients. Recall that the amplitude of the non-linearities of $\bar{D}_{j}$ decreases with increasing $\mu$. Friction promotes disorder and makes it more difficult for beads to arrange regularly, which smoothes the variations of $\bar{D}_{j}$ due to the ring effect.

\section{Jamming in bidisperse bead assemblies}

Bidisperse media are characterized by two distinct bead diameters $\Phi_{1}$ and $\Phi_{2}$. We gather them into one single parameter, the volume-average diameter $\bar{\Phi}$ of the beads:

$$
\bar{\Phi}=\frac{N_{1} \Phi_{1}^{4}+N_{2} \Phi_{2}^{4}}{N_{1} \Phi_{1}^{3}+N_{2} \Phi_{2}^{3}},
$$

where $N_{1}$ and $N_{2}$ are the number of beads with diameters $\Phi_{1}$ and $\Phi_{2}$, respectively. Measures of the median jamming diameter have been performed for bead assemblies with $\mu=0.2$ and $\bar{\Phi}$ ranging from $3.0 \mathrm{~mm}$ to $6.0 \mathrm{~mm}$. The resulting median jamming diameters are plotted against $\bar{\Phi}$ in Figure 6 (top curve) together with the monodisperse results obtained with $\mu=0.2$. In this representation, bidisperse measures collapse on the monodisperse curve with very good accuracy. One has to be cautious though, for bidisperse media with $\Phi_{1}$ significantly smaller than $\Phi_{2}$, yet close enough to $\bar{\Phi}$ like the measures obtained for $\bar{\Phi}=3.25 \mathrm{~mm}$ and $\bar{\Phi}=3.75 \mathrm{~mm}$ with the $\operatorname{mix} \Phi_{1}=3.0 \mathrm{~mm}$, $\Phi_{2}=6.0 \mathrm{~mm}$. In those cases, the median jamming diameters are statistically greater for bidisperse media than for monodisperse ones. This can be explained in terms of segregation. Indeed, such granular assemblies feature a small number of large beads that are significantly larger than the others. This leads to a strong tendency to segregate while the medium flows. Large beads actually migrate to the top and remain there while small beads flow through the hole. When the large beads finally reach the hole, most of the time they gather in an arch.

Otherwise, bidisperse and monodisperse results fit very well for $\mu=0.2$. This comes all the more unexpected since a monodisperse bead assembly defined using the volume-average diameter does not fill the same volume fraction as the corresponding bidisperse assembly. One can further observe that two bidisperse media with same $\bar{\Phi}$ but different diameters for their constituting beads will have the same median jamming diameter. Those observations lead us to formulate the following tentative law:

For fixed interparticulate friction, the median jamming diameter of spatially homogeneous bead assemblies only depends on their volume-average diameter.

With frictionless beads, the dependence of $\bar{D}_{j}$ on the diameter of the beads is strongly non-linear due to the influence of bead rings. Figure 6 (lower curve) reports results of both monodisperse and bidisperse trials obtained with $\mu=0.0$ and $\bar{\Phi}$ ranging from $4.5 \mathrm{~mm}$ to $7.0 \mathrm{~mm}$. One 
can see that only few bidisperse values of $\bar{D}_{j}$ lie on the line $\tau_{0.0}$, suggesting that the ring effect indeed disappears in bidisperse assemblies, but only for particular mix configurations.

First observe that all bidisperse assemblies with $\Phi_{2}=$ $7.0 \mathrm{~mm}$ have identical behaviors when $\bar{\Phi}$ is close to $7.0 \mathrm{~mm}$ : their median jamming diameter is nearly constant for $\bar{\Phi}$ between $5.75 \mathrm{~mm}$ and $6.75 \mathrm{~mm}$ and then significantly increases until it joins up with the monodisperse measures at $\bar{\Phi}=7.0 \mathrm{~mm}$. These variations recall those we observe with monodisperse assemblies and this is because such mixes are nearly monodisperse and still generate bead rings. In this case, however, the ring diameters $\delta_{i}$ do not depend on $\bar{\Phi}$, but only on $\Phi_{2}$ : for a given value of $\Phi_{2}$ the $\delta_{i}$ will be equal to those obtained with monodisperse assemblies whose beads have diameters $\Phi=\Phi_{2}$.

In the monodisperse case with $\Phi=7.0 \mathrm{~mm}$, the bead rings have diameters $\delta_{1}=43.0 \mathrm{~mm}, \delta_{2}=29.96 \mathrm{~mm}$ and $\delta_{3}=16.52 \mathrm{~mm}$ (see Fig. 5). As discussed in Section 5, $\bar{D}_{j}$ tends to stay between two ring diameters. For bidisperse assemblies with $\Phi_{2}=7.0 \mathrm{~mm}$ and $\bar{\Phi}$ close to $\Phi_{2}$ (that is $\bar{\Phi} \geq 5.75 \mathrm{~mm}$ ), $\bar{D}_{j}$ then tends to stay below $\delta_{3}$ which explains why it remains constant for $5.75 \mathrm{~mm} \leq$ $\bar{\Phi} \leq 6.75 \mathrm{~mm}$. Here, $\bar{D}_{j}$ follows the constant behavior of the $\delta_{i}$ for the same reasons it follows their decreasing behavior in the monodisperse case.

Observe, though, that for $\bar{\Phi} \geq 5.75 \mathrm{~mm}$, all values of $\bar{D}_{j}$ measured on bidisperse assemblies with $\Phi_{2}=7.0 \mathrm{~mm}$ collapse on the same curve. Now, consider the measures of $\bar{D}_{j}$ obtained with mixtures $\Phi_{1}=5.0 \mathrm{~mm}, \Phi_{2}=6.0 \mathrm{~mm}$ and $\Phi_{1}=5.0 \mathrm{~mm}, \Phi_{2}=7.0 \mathrm{~mm}$ (Fig. 6). For $\bar{\Phi} \leq 5.75 \mathrm{~mm}$, one can see that they collapse on the same curve as well. Further observe that mixtures with $\Phi_{1}=5.0$ are in analogous conditions regarding the bead rings when $\bar{\Phi}$ is close to $5.0 \mathrm{~mm}$. This suggests that our law remains valid for bead assemblies submitted to the ring effect, provided that they are in identical conditions regarding ring formation.

Finally, one can see that the values of $\bar{D}_{j}$ obtained with the mixture $\Phi_{1}=4.0 \mathrm{~mm}, \Phi_{2}=7.0 \mathrm{~mm}$ collapse on $\tau_{0.0}$ for $4.9 \mathrm{~mm} \leq \bar{\Phi} \leq 5.2 \mathrm{~mm}$. Indeed, $\bar{\Phi}$ is away from both $\Phi_{1}$ and $\Phi_{2}$, and the corresponding assemblies are bidisperse enough to prevent ring formation.

Similarly to friction, the degree of polydispersity is a parameter that induces disorder in granular assemblies and makes it more difficult for such regular patterns as bead rings to build. When friction disappears, the degree of polydispersity becomes predominant in this role, and regulates the variations of $\bar{D}_{j}$ as just discussed.

\section{Experimental validation}

In order to check the law formulated above, experiments with bidisperse sets of real beads were carried out. While it is easy to perform monodisperse experiments, bidisperse ones turn out to be a lot trickier. Indeed, strong segregation can occur when pouring beads of two distinct diameters into the initial piling of a trial and when the medium flows, it behaves like a succession of more or less thick monodisperse layers. Thus, much care is needed

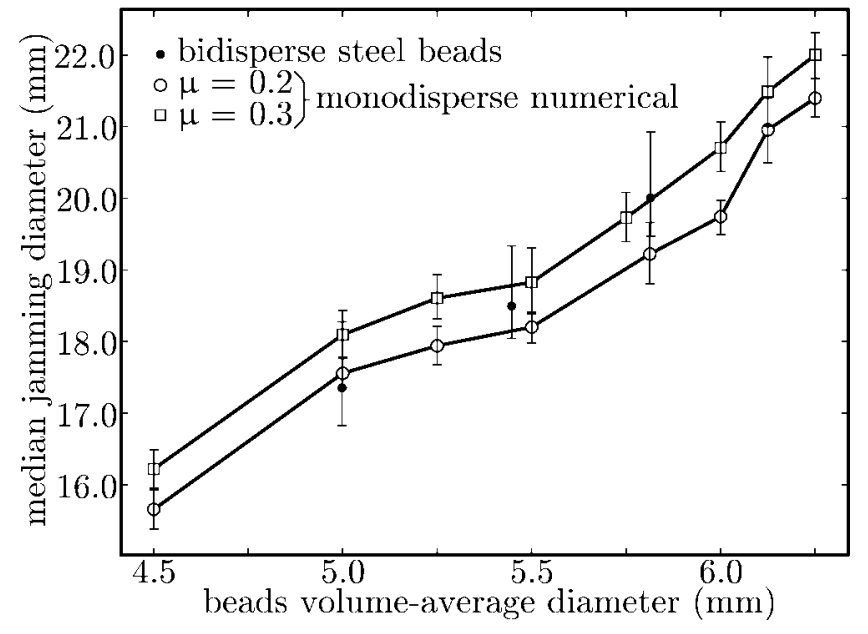

Fig. 7. Median jamming diameters obtained numerically and experimentally for $4.5 \mathrm{~mm} \leq \bar{\Phi} \leq 6.25 \mathrm{~mm}$. Experimental results are obtained using bidisperse assemblies of steel beads with $\Phi_{1}=4.0 \mathrm{~mm}$ and $\Phi_{2}=7.0 \mathrm{~mm}$. Numerical results are obtained using monodisperse assemblies with friction coefficients $\mu=0.2$ and $\mu=0.3$.

Table 2. Results of experimental trials on bidisperse sets of steel beads with $\Phi_{1}=4.0 \mathrm{~mm}$ and $\Phi_{2}=7.0 \mathrm{~mm}$.

\begin{tabular}{cc}
\hline $\bar{\Phi}(\mathrm{mm})$ & $\bar{D}_{j}(\mathrm{~mm})$ \\
\hline 5.00 & $17.35 \pm 0.93$ \\
5.45 & $18.50 \pm 0.84$ \\
5.81 & $20.01 \pm 0.93$ \\
\hline
\end{tabular}

when performing physical experiments. We use the following procedure: small bidisperse subsets of between 10 and 20 beads with the wanted volume-average diameter $\bar{\Phi}$ are poured one after the other into the container. The latter is turned around its vertical axis by hundred twenty degrees between two consecutive pourings. The beads are poured gently to avoid that they mix with those that have already settled below. Preparing one such homogeneous bidisperse bead assembly takes approximately one hour.

The diameters of the steel beads constituting our experimental bidisperse media are $\Phi_{1}=4.0 \mathrm{~mm}$ and $\Phi_{2}=7.0 \mathrm{~mm}$ and three volume-average diameters $\bar{\Phi}$ have been investigated: $5.0 \mathrm{~mm}, 5.45 \mathrm{~mm}$, and $5.81 \mathrm{~mm}$. Table 2 reports the median jamming diameters measured using those experiments. Comparing Tables 1 and 2, one can see that monodisperse steel bead assemblies with $\Phi=5.0 \mathrm{~mm}$ and bidisperse steel bead assemblies with volume-average diameter $\bar{\Phi}=5.0 \mathrm{~mm}$ have nearly identical median jamming diameters. This is a first experimental confirmation of our law.

The values of $\bar{D}_{j}$ listed in Table 2 are plotted in Figure 7 against $\bar{\Phi}$, together with measures obtained numerically on monodisperse media with friction coefficients $\mu=0.2$ and $\mu=0.3$. One can see that experimental points lie close to both numerical curves. As discussed in Section 4, monodisperse results suggest that the numerical friction coefficient $\mu$ corresponding to our steel 
beads should lie between 0.2 and 0.3 . This is confirmed by Figure 7 and indicates that our simulation model is indeed able to reproduce static granular media. Moreover, the good agreement observed in this figure between the experimental bidisperse results and the numerical monodisperse ones provides a further validation of our tentative law.

\section{Conclusion}

Our experimental and numerical trials have resulted in an excellent agreement of the corresponding estimated median jamming diameters, thereby validating numerical simulation as an appropriate tool for the study of jamming. Furthermore, we have investigated the role of friction and boundary conditions on the median jamming diameter as a function of bead diameters and identified the formation of bead rings as an important element therein. Finally we have studied bidisperse media and found (both by simulation and experiment) their jamming behavior to be characterized by their volume-average diameter over a wide range of granulometries. The following questions remain open for future research: using our approach for experimental determination of the bead-bead friction coefficient, finding the influence of orifice and container shapes on jamming, and studying the dynamic interplay between segregation and jamming in order to refine the empirical law we formulate here.

This project was partially funded by the Swiss National Science Foundation, grant No. 200020-100499/1 and by armasuisse. Many thanks to J. Bahar and S. Hold for carrying out the bidisperse experiments and to M.-O. Boldi for his statistical guidance. Finally we want to express our appreciation for the insightful and stimulating remarks by the two referees.

\section{References}

1. W.A. Beverloo, H.A. Leniger, J. Van De Velde, Chem. Eng. Sci. 15, 260 (1961).

2. R.L. Brown, J.C. Richards, Principles of Powder Mechanics (Pergamon Press, 1970).

3. I. Zuriguel, L.A. Pugnaloni, A. Garcimartin, D. Maza, Phys. Rev. E 68, 030301 (2003).

4. I. Zuriguel, A. Garcimartin, D. Maza, L.A. Pugnaloni, J.M. Pastor, Phys. Rev. E 71, 051303 (2005).

5. R. Arévalo, D. Maza, L.A. Pugnaloni, Phys. Rev. E 74, 021303 (2006).

6. L.A. Pugnaloni, M.G. Valluzzi, L.G. Valluzzi, Phys. Rev. E 73, 051302 (2006).

7. L.A. Pugnaloni, G.C. Barker, Physica A 337, 428 (2004).

8. P.A. Cundall, O.D.L. Strack, Géotechnique 29, 47 (1979).

9. J.-A. Ferrez, Th.M. Liebling, Philos. Mag. B 82, 905 (2002).

10. L. Pournin, A. Mocellin, Th.M. Liebling, Phys. Rev. E 65, 011302 (2002).

11. L. Pournin, M. Weber, M. Tsukahara, J.-A. Ferrez, M. Ramaioli, Th.M. Liebling, Granular Matter 7, 119 (2005).

12. K. Bagi, Granular Matter 7, 31 (2005).

13. A.C. Davison, D.V. Hinkley, Bootstrap Methods and Their Application (Cambridge University Press, 1997).

14. A. Gioia, Pharm. Technol., February 1980, p. 65.

15. G. Enstad, Chem. Eng. Sci. 30, 1273 (1975).

16. A.J. Matchett, Powder Technol. 171, 133 (2007).

17. K. To, P.-Y. Lai, H.K. Pak, Phys. Rev. Lett. 86, 71 (2001).

18. N.A. Pohlman, B.L. Severson, J.M. Ottino, R.M. Lueptow, Phys. Rev. E 73, 031304 (2006). 